\title{
Nano-archaeometry or nano-archaeological Science.
}

\author{
Gianni Gallello \\ 1 University of York
}

The development of miniaturised analytical methods to be applied for archaeological or culture heritage material studies. At the moment this area of research is just starting to be developed. The need to minimise the impact in unique remains and use powerful and sensitive analytical methods is improving the research at nanoscale level. 